\title{
The Ocean Biogeographic Information System (OBIS): an on-line, worldwide atlas for accessing, modeling and mapping marine biological data in a multidimensional geographic context
}

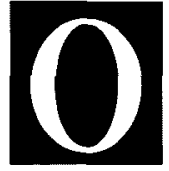

BIS is a component of the Census of Marine Life (CoML) an international program to assess and explain the diversity, distribution, and abundance of marine life. There is no adequate system for retrieval of ocean biological data. The few existing databases do not usefully summarize known distributions and abundance of marine life nor are they organized to encourage frequent use and intercomparison of datasets. An on-line, user-friendly system for absorbing, integrating, and assessing data about life in the oceans will stimulate taxonomic and systematic research and generate new hypotheses concerning evolutionary processes, factors related to maintenance of species distributions, and roles of marine organisms in marine ecosystem function.

Over the past three decades there have been major advances in understanding the outlines of relationships among broadly-defined trophic units and their biogeochemical roles in ecosystems. Understanding of spatial pattern of marine ecosystems, their evolution, and how they respond to environmental change will require greater use of species-level data. The geographical boundaries of ecosystems are poorly defined and there is little agreement on the number of faunal provinces and their boundaries. The United Nations Environmental Program Global Biodiversity Assessment (UNEP, 1995) provides two biogeographic maps: one recognizes six classes of "Oceanic Realms" (p. 100) and the other classifies coastal fisheries management areas into 49 "Large Marine Ecosystems" (p.
502). A more recent global classification of surface waters recognizes 51 geographic provinces (Longhurst, 1998). Accurate data on the spatial and temporal distribution of most marine species are not readily available. There are no good maps of marine biodiversity (Grassle and Stocks, 1999) and biogeographic classifications seldom consider life in deep-sea sediments where communities have high marine biodiversity. Description of previously unknown species and higher taxa of marine life from both deep-sea and shallow bottom communities continues steadily and, in the case of some groups, at an increasing pace (Figure 1). As accurate data on abundance and distribution of marine taxa accumulate, there is an urgent need for information systems to retrieve and analyze data in the context of temporal and spatial patterns of synoptic physical and biological data from satellites, improved bathymetry, and output from global models.

In the past two decades, oceanography has become a more mature, integrative, interdisciplinary science. The roles of major taxa in chemical and geological processes in the ocean are becoming better understood and the field of biogeochemical research has grown rapidly. Biologists and physical oceanographers are working together to model interactions of taxonomic and functional groups of organisms amongst each other and with the physical environment. Such coupled physi$\mathrm{cal} /$ biological models increase the resolution of biological elements to provide greater realism. In parallel, greater integration within the biological sciences is 


\section{Species Descriptions}

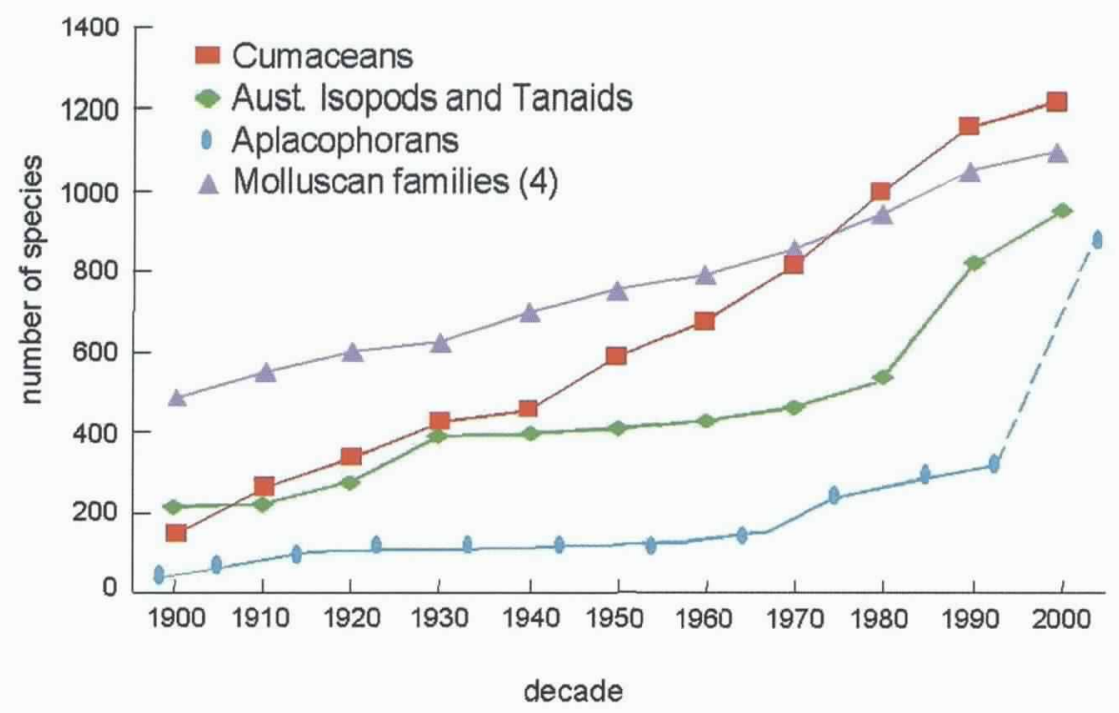

Figure 1. Number of species described for selected taxa from 1900 to the present. The data for four molluscan families have been replotted from Bouchet (1997). The other three data sets were presented at an October 1997 Benthos Census Workshop sponsored by the Alfred P. Sloan Foundation at Rutgers University: Australian isopods and tanaids, G.C.B. Poore; Cumaceans, L. Watling; and aplacophoran molluscs, A. Scheltema.

reflected in new programs to study biocomplexity and use of the term "integrative biology" to describe some academic departments and a scientific journal.

The trend toward integration of biological research is stimulated by the rapid growth in the application of molecular techniques to a broad range of biological processes. Species are the basic units of biological organization and evolutionary change. Species definitions now include characteristics of the genome using a variety of molecular technologies, morphological description based on new tools for identification, abundance data, as well as the traditionally-used data on geographical location of collecting sites. Molecular genetics data useful in defining species can also be analyzed to indicate evolutionary relationships among species (systematics), variations in the temporal and spatial scales of contemporary species interactions, relationships between species assemblages and the environment (ecology), and, by study of specific enzymes, their relative contribution to ecosystem function (biogeochemistry). A system for retrieval and analysis of geo-referenced data from all levels of biological organization in the context of data describing the full range of oceanographic processes is required.

The success of OBIS will be measured by its ability to store, search, and retrieve data; to analyze the spatial and temporal organization of new biological information in a four-dimensional map-based environmental context; and to suggest previously unobserved ecological and evolutionary relationships. The present record of life in the ocean is mostly based on collections in museums and scientific publications that provide lists of species names, location (latitude, longitude, and depth), sometimes numbers or biomass, and usually something about how they were collected. These names and numbers are a pale reflection of reality but, to an experienced marine biologist, these data describe a rich world of plants and animals interacting amongst themselves and their environment. Coral reef data may evoke the experience of swimming over a reef edge surrounded by a plethora of brightly-colored fish. Data from a deep-sea mud sample recall months looking at small invertebrates under a microscope for a glimpse at one of the most species-rich environments on the planet. A sample from Buzzards Bay in Massachusetts yields a rare crustacean near the base of an evolutionary tree leading to shrimp and crabs. Counts and names of worms and clams evoke porthole views from submersible dives to deep-sea hydrothermal vents. Every year, optical or acoustic imaging devices used on every imaginable platform from submersibles to satellites provide broader coverage and more highly resolved representations of life in the ocean. Despite these advances, even the best images do not provide a systematic, quantitative representation of the distribution of and relationships among organisms and how this living environment changes with time. OBIS will make it possible to conjure an image of the environment through relationships among datasets, including databases of images themselves. To provide quality control for OBIS as methodologies change and improve, museum specimens will be even more important as reference material for comparison.

Many of the papers in this issue originated at an international Workshop on the Ocean Biogeographical Information System held in Washington, D.C., 3-4 November 1999. Names of attendees, organizations represented, and a brief summary can be found on the Consortium for Oceanographic Research and Education web site (http://core.cast.msstate.edu/censobis1.html). After considerable discussion, participants defined OBIS as: "An on-line world-wide marine atlas 'infrastructure' providing scientists with the capability of operating in a four-dimensional environment so that analyses, modeling, and mapping can be accomplished in response to user demand through accessing and providing relevant data." Emphasis was placed on interoperability through common definition of metadata standards and protocols for the distributed, multi-tiered architecture of OBIS. It was agreed that OBIS would be managed as a federation of database sources that reach agreement on the means to achieve interoperability, yet 
allow a high degree of autonomy with respect to both existing and developing data systems. This OBIS federation will be governed by an international Steering Committee and an OBIS Program Office will provide management, communications, and networking support for members. OBIS will make geographicallyreferenced information on distribution and abundance of species readily available, provide linkages to genomic databases of DNA type sequences, indicate location of reference specimens, and facilitate use of analytical, mapping, and modeling tools. The rescue and placement in an accessible digital format of existing databases will receive high priority. To facilitate the development of new hypotheses about factors controlling the distribution and abundance of marine life, the OBIS network will: 1) provide access to global, synoptic environmental data from satellites, 2) data from in situ measuring systems, and 3) relevant geo-referenced products from physical and chemical models. Global coverage of data on variables such as primary production, export production, phytoplankton taxa distinguishable from satellite images, temporal patterns of temperature variation, river discharge, currents, and near-bottom kinetic energy are examples of datasets potentially important in studying biogeographic patterns of distribution. The presentation and analysis of co-occurrence data on distribution and abundance of species in the context of environmental variables will help revitalize the study of marine taxonomy, systematics, and biogeography.

In May 2000, the U.S. Government Agencies in the National Ocean Partnership Program together with the Alfred P. Sloan Foundation announced a set of 8 grants to initiate OBIS (http://core.cast.msstate.edu/censpr1.html). The grants involve researchers in more than 60 institutions in 15 countries, and address overall system architecture as well as a range of taxonomic groups including fishes, bivalves, gastropods, cephalopods, calanoid copepods, euphausiids, anemones, corals, and several gelatinous zooplankton taxa. The National Ocean Partnership Program is expected to consider additional OBIS-related proposals this year.

The organization of OBIS will require continued meetings with representatives of international database organizations such as GBIF, FAO, UNESCO-IOC Register of Marine Organisms, Species 2000, ETI, Gaia 21, GenBank, Zoological Record, FishBase, CephBase, FISHNET, European Register of Marine Organisms (ERMS), and representatives of national biodiversity or oceanographic data centers. At the end of 2000, the OBIS Steering Committee and plans for the Program
Office will be formed and, following recommendations from the Workshop, its first tasks will be:

- To develop a metadata catalog of sources of primary data

- To agree on minimum metadata standards and protocols

- To support mechanisms to assure accuracy of taxonomic identifications and common agreement on systematic relationships

- To agree on a strategy for data discovery, setting of priorities for data rescue, and assessment of data quality, and an international network of taxonomic authorities to resolve nomenclature issues

- To agree on the issue of intellectual property rights

Consonant with widespread interest in understanding the biocomplexity of natural systems, the Census of Marine Life is planning research programs to consider oceanic biology from genes to ecosystems at time and space scales requiring use of new technologies. Simultaneously a rapidly-developing Global Ocean Observing System is already providing a continuous stream of physical observations to challenge existing means for data access, analysis, and presentation. In the Internet Age, new strategies for deploying this information and knowledge must evolve. The papers in this issue demonstrate that ideas and willingness abound to create the Ocean Biogeographic Information System. The biggest challenge is for the various stakeholders worldwide in marine biological data to learn quickly to work together.

\section{Acknowledgements:}

I thank the November, 1999 OBIS Workshop participants and particularly Karen Stocks and Yunqing Zhang for their contributions.

\section{REFERENCES:}

Bouchet, P. 1997: Inventorying the molluscan diversity of the world: what is our rate of progress? The Veliger, $40,1-11$.

Grassle, J.F. and K.I. Stocks, 1999: A Global Ocean Biogeographic Information System (OBIS) for the Census of Marine Life. Oceanography, 12(3), 12-14.

Longhurst, A., 1998: Ecological Geography of the Sea. Academic Press, San Diego.

UNEP, United Nations Environmental Programme, 1995: Global Biodiversity Assessment. V. H. Heywood, Ed., R.T. Watson, Chair, Cambridge University Press, Cambridge. 